\title{
Differential Scattering Cross-Sections for the Different Product Vibrational States in the Ion-Molecule Reaction $\mathrm{Ar}^{+}+\mathrm{N}_{2}$
}

\author{
S. Trippel,${ }^{1}$ M. Stei, ${ }^{2}$ J. A. Cox,${ }^{3}$ and R. Wester ${ }^{2}, *$ \\ ${ }^{1}$ Center for Free-Electron Laser Science, DESY, Notke-Straße 85, 22706 Hamburg, Germany \\ ${ }^{2}$ Institut für Ionenphysik und Angewandte Physik, Universität Innsbruck, Technikerstraße 25, 6020 Innsbruck, Austria \\ ${ }^{3}$ Department of Plant Sciences, University of Cambridge, Downing Street, Cambridge CB2 3EA, United Kingdom
}

(Received 14 December 2012; published 15 April 2013)

\begin{abstract}
The charge transfer reaction $\mathrm{Ar}^{+}+\mathrm{N}_{2} \rightarrow \mathrm{Ar}+\mathrm{N}_{2}^{+}$has been investigated in a crossed-beam experiment in combination with three-dimensional velocity map imaging. Angular-differential state-to-state cross sections were determined as a function of the collision energy. We found that scattering into the first excited vibrational level dominates as expected, but only for scattering in the forward direction. Higher vibrational excitations up to $v^{\prime}=6$ have been observed for larger scattering angles. For decreasing collision energy, scattering into higher scattering angles becomes increasingly important for all kinematically allowed quantum states. Our detailed measurements indicate that a quantitative agreement between experiment and theory for this basic ion-molecule reaction now comes within reach.
\end{abstract}

PACS numbers: $34.50 .-\mathrm{s}, 34.70 .+\mathrm{e}, 82.20 . \mathrm{Bc}, 82.30 . \mathrm{Fi}$

Gas phase studies of ion-molecule reactions have provided insight into a multitude of chemical processes in environments where ions and neutrals coexist. Ionmolecule reactions determine the abundance of many of the complex species that can be detected in interstellar molecular clouds and in planetary atmospheres [1,2]. The conceptually simple charge transfer reactions are particularly interesting, as they were found to explain the x-ray emission from comets $[3,4]$ and may serve as a possible acceleration mechanism of cosmic rays due to strong shock waves in supernova remnants [5]. Charge transfer is also of cosmological importance in that it shapes the hydrogen chemistry in the early universe [6]. Laboratory measurements and theoretical calculations are needed to provide the basis for modeling these processes. In addition, charge transfer reactions lead to characteristic light emission from excited states that are useful to determine the parameters of laboratory or technical plasmas, such as temperature, velocity, electron density, and charge states of ions $[7,8]$. To reach very low collision energies, charge transfer has been studied near threshold in half collisions [9]. Recently, ultracold charge transfer reactions have become of interest in studies of cold atom-ion collisions, which are carried out to investigate quantum mechanical phenomena in scattering processes at very low energies [10-12].

Charge transfer reactions in gas phase evolve in many cases not on a single potential energy surface and are therefore often accompanied by a breakdown of the BornOppenheimer approximation. Because the shape of the molecule changes upon charge transfer, state-to-state electron transfer rates are often controlled by intramolecular vibrational motion [13-15]. While in many cases the outcome of a reaction at high collision energies is well described by the properties of the isolated molecule, the results at low energies are not explained by a Frank-Condon treatment $[16,17]$. This behavior may be explained by molecular bond distortions due to the electric field of the incoming ion [18] and to short range repulsive interactions between the projectile and the target [19].

A model system of a gas phase non-BornOppenheimer reaction mechanism is the charge transfer reaction

$$
\operatorname{Ar}^{+}\left({ }^{2} \mathrm{P}_{J}\right)+\mathrm{N}_{2}\left({ }^{1} \Sigma_{g}^{+}, v=0\right) \rightarrow \operatorname{Ar}\left({ }^{1} \mathrm{~S}_{0}\right)+\mathrm{N}_{2}^{+}\left({ }^{2} \Sigma_{g}^{+}, v^{\prime}\right) .
$$

Despite its overall exoergicity the most abundant product channel at low collision energy is $\mathrm{N}_{2}^{+}$in the first vibrationally excited level, which is endoergic by $0.092 \mathrm{eV}$. Experimental evidence was obtained early on that this channel is formed despite having a much lower FranckCondon factor than the exoergic ground state [20,21]. Later, angle-differential cross sections and vibrational and rotational state distribution have been determined in the energy range of $0.3-3 \mathrm{eV}$ [17,22-26]. Here, crossedbeam experiments [25] found much higher vibrational excitation in the collision energy range near $1 \mathrm{eV}$ collision energy than radiofrequency ion guide experiments [23]. Even after reconsidering the finite velocity and angular resolution and the involved unfolding scheme of the crossed-beam experiments, this disagreement remained unresolved [27]. In addition, state-of-the-art theoretical calculations could not achieve an agreement with the measured product energy and angular distributions [28-31]. More recently, a semiclassical Landau-Zener model has been employed to derive state-to-state cross sections $[16,32]$. In this model higher vibrational excitations are predicted for larger scattering angles. When we developed the crossed-beam spectrometer for ion-molecule reactions based on the velocity map imaging technique [33], we revisited the reaction of $\mathrm{Ar}^{+}$with $\mathrm{N}_{2}$ and found again 
significant vibrational excitation, but still with insufficient resolution to provide a clear picture of the reaction [34].

In this Letter we present detailed angular-differential scattering cross sections together with a product vibrational state analysis for the $\mathrm{Ar}^{+}+\mathrm{N}_{2}$ charge transfer reaction. Using an optimized design of the velocity map imaging potentials with respect to stray electric fields and a full three-dimensional measurement of the product velocity vectors, we are now able to assign the different vibrational states of the $\mathrm{N}_{2}$ product ions in the measured kinetic energy distributions. Our results clearly disagree with the previous crossed-beam cross section measurements [25], possibly caused by the difficulty to unambiguously unfold the previous angle-resolved scattering data. Our measured angular distributions change systematically with product vibrational state, qualitatively in agreement with semiclassical calculations $[16,32]$.

The experiment is based on our previous work on negative ion-molecule reactions $[34,35]$. Crossed-beam imaging studies of ion-molecule reactions have recently also been reported for reactions of $\mathrm{C}^{+}$with $\mathrm{NH}_{3}$ [36]. Here, $\mathrm{Ar}^{+}$ions are produced by a combination of an electron gun and a supersonic Ar gas pulse provided by a piezoelectric valve. In the extraction volume of a Wiley McLaren mass spectrometer the ions are extracted perpendicularly and accelerated toward the crossed-beam spectrometer. Prior to collision the ions are decelerated inside a cylindrical electrode just outside the spectrometer to the desired kinetic energies in the range of $1.0-5.6 \mathrm{eV}$, which are measured with the velocity map imaging spectrometer (FWHM of about $200 \mathrm{meV}$ ). $\mathrm{Ar}^{+}$may generally be produced in the $P_{1 / 2}$ and $P_{3 / 2}$ states by electron impact ionization. As discussed below, we estimate a contribution of less than $20 \%$ of the $P_{1 / 2}$ state in this experiment. The $\mathrm{N}_{2}$ target beam is generated in a pulsed supersonic expansion, provided by a piezoelectric valve with stagnation pressure of 2 bar at a temperature of $70^{\circ} \mathrm{C}$. The central part of the supersonic beam enters the scattering center after passing a skimmer with an orifice diameter of $200 \mu \mathrm{m}$, placed $35 \mathrm{~mm}$ behind the nozzle. For $\mathrm{N}_{2}$ we expect translational and rotational temperatures of about $5 \mathrm{~K}$ [37]. The laboratory velocity of the molecules is measured after electron impact ionization with the velocity map imaging setup to be around $830 \mathrm{~m} / \mathrm{s}$, in good agreement with expectation.

The ion and the molecular beam cross each other in the center of a velocity map imaging stack at a scattering angle of $61^{\circ}$. Once the two reactant pulses have crossed, the velocity map imaging electrodes are switched on to map the ion velocities onto the imaging detector, which consists of a microchannel plate combined with a phosphor screen and a CCD camera. The ion time of flight is obtained by a photomultiplier tube in combination with a time-to-digital converter picking up the rising edge of the light spot on the phosphor screen. Typical event rates are less than one ion per bunch crossing with a background rate about 2 orders of magnitude lower. Images with two or more detected ions were neglected to be able to correlate transverse position and time of flight. Ion-impact positions and time-of-flight information are used to determine the three-dimensional velocity vector of the ions in the interaction region. Note that the velocity vector is measured irrespective of the scattering angle, yielding an effective $4 \pi$ angular acceptance. In order to suppress signals from the $\mathrm{Ar}^{+}$ reactant beam, the detector is activated for a period of $1 \mu \mathrm{s}$ around the $\mathrm{N}_{2}^{+}$arrival time.

We have measured the differential cross section of reaction for relative collision energies of $0.3,0.5,0.8$, $1.2,1.7$, and $2 \mathrm{eV}$. A representative histogram of the cross section at $1.7 \mathrm{eV}$ is shown in Fig. 1(a). It is obtained by determining the three-dimensional velocity vector for each $\mathrm{N}_{2}$ product ion in the center-of-mass frame and computing its components in the scattering plane. In order to compare our results with slice and projected images used in previous measurements, each velocity entry is weighed by $1 / v_{\perp}$ where $v_{\perp}$ is the velocity component perpendicular to the symmetry axis, i.e., the relative velocity axis of the scattering process. For the same reason we also extend the image by mirroring the data to negative values of $v_{\perp}$. The $\mathrm{Ar}^{+}$reactant velocity is indicated by an arrow pointing along the positive $v_{\|}$axis and the $\mathrm{N}_{2}$ educt velocity is indicated by an arrow pointing along the negative $v_{\|}$axis. Newton rings mark the product velocities corresponding to $v^{\prime}=0-4$ vibrational quanta in the $\mathrm{N}_{2}$ product ion. A sharp peak in the forward direction is observed in the differential cross section. Additionally, a contribution of larger scattering angles in the forward hemisphere is found. The forward scattering peak shows product velocities mainly corresponding to $v^{\prime}=1$. The sideways scattered products clearly show vibrational excitation in higher $v^{\prime}$ states.

For a quantitative analysis, the angular distributions of each product vibrational state are extracted from the measured cross section images. Figure 1(b) shows the internal energy distribution of the product $\mathrm{N}_{2}^{+}$ions scattered into the narrow cone of forward scattering angles $\theta=5 \pm 5^{\circ}$ [marked in black in Fig. 1(a)]. Red lines indicate the internal energy of $\mathrm{N}_{2}^{+}$in the vibrational levels $v^{\prime}=1-7$. As expected, most of the product ions are excited with one vibrational quantum in agreement with previous measurements and theoretical predictions. There is only a small contribution of the levels with $v^{\prime}=0,2$, and 3. In addition, we estimate from the shown distribution a contribution from reactions of the $P_{1 / 2}$ state of $\mathrm{Ar}^{+}$of less than $20 \%$. Otherwise a strong peak at $0.36 \mathrm{eV}$ internal energy would have to be present in the distribution. Such a small contribution is in accord with a statistical mixture of $P_{3 / 2}: P_{1 / 2}$ of 2:1 and the lower reactivity of $\operatorname{Ar}^{+}\left(P_{1 / 2}\right)$ [38].

The internal energy distribution of product ions scattered into $\theta=45 \pm 5^{\circ}$ [also marked in black in Fig. 1(a)] is shown in Fig. 1(c). For these scattering angles $v^{\prime}=1$ is 



FIG. 1 (color online). (a) Differential scattering cross section for the reactive scattering of $\mathrm{Ar}^{+}+\mathrm{N}_{2}$ at a relative energy of $1.7 \mathrm{eV}$. The image is obtained by rotating and weighting the measured three-dimensional velocity distribution of $\mathrm{N}_{2}^{+}$ions. Reactant velocities are indicated by black arrows $\left(\mathrm{N}_{2}\right.$ along the negative and $\mathrm{Ar}^{+}$along the positive $v_{\|}$axis). Newton spheres are plotted which correspond to $\mathrm{N}_{2}^{+}$vibrational excitation with $v^{\prime}=0-4$. Black lines indicate cuts on the scattering angle $\theta$. (b) $\mathrm{N}_{2}^{+}$energy distribution with a cut on the scattering angle $\theta=5 \pm 5^{\circ}$. (c) $\mathrm{N}_{2}^{+}$energy distribution with a cut on the scattering angle $\theta=45 \pm 5^{\circ}$. Blue lines in panels (b),(c) are fits to a sum of Gaussian, each centered at $v^{\prime}=0-7$.

not the dominant product channel anymore. Instead, the internal energy distribution exhibits higher vibrational excitations with the majority of ions being scattered into $v^{\prime}=2$. It can be seen from Figs. 1(b) and 1(c) that the individual product vibrational levels are not resolved. This can be attributed to the experimental energy resolution dominated by the finite energy width of the slow ion beam. The expected resolution depends on the product scattering angle and is calculated to change from $0.06 \mathrm{eV}$ for forward scattering to $0.2 \mathrm{eV}$ for backward scattering. A second contribution to the measured broadening stems
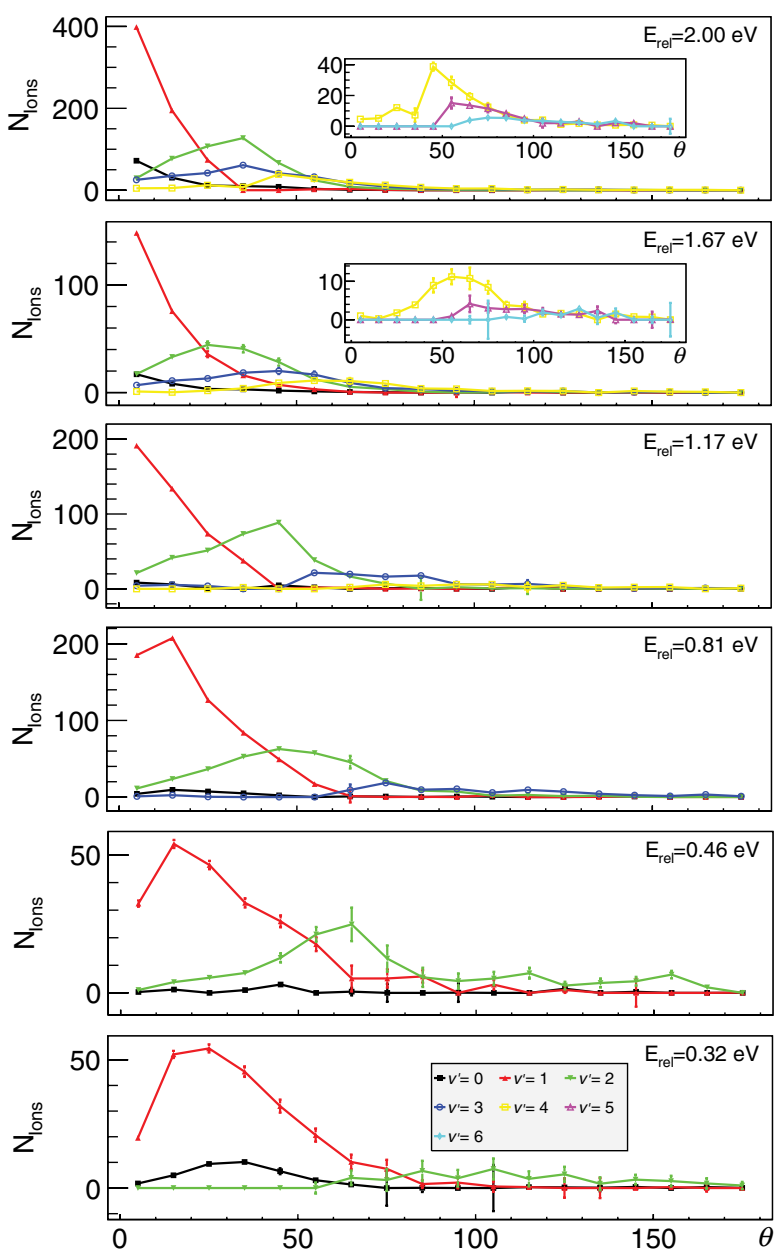

FIG. 2 (color online). Number of ions scattered into vibrational state $v^{\prime}=0-6$ of the $\mathrm{N}_{2}^{+}$product ion as a function of scattering angle $\theta$ for all measured energies between 0.3 and $2 \mathrm{eV}$. Scattering into $v^{\prime}=1$ dominates for small scattering angles whereas higher vibrational excitation is more pronounced at larger angles. All kinematically allowed product levels are scattered into increasing larger angles with decreasing collisional energy.

from rotational excitation of $\mathrm{N}_{2}^{+}$, which is on the order of $0.06 \mathrm{eV}[17,26]$.

To analyze the angular dependence of the scattering cross section for each vibrational level separately, the relative vibrational populations are extracted from the measured energy distributions for $10^{\circ}$ scattering-angle intervals between 0 and $180^{\circ}$. For this we fit a sum of Gaussian functions, each representing a different vibrational level $v^{\prime}$, to the energy distributions for each scattering angle interval. Only the heights of the Gaussian functions are free fit parameters, the mean of each Gaussian is predetermined by the vibrational excitation energy of each level $v^{\prime}$. The Gaussian widths represent the product energy resolution and are calculated from the measured energy resolution of the reactant beams. The width is assumed to be the same for all Gaussians in a 


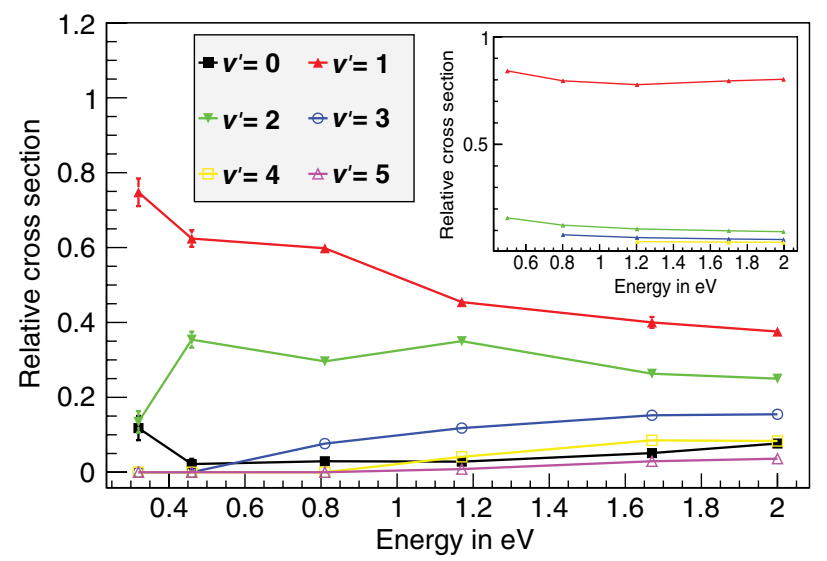

FIG. 3 (color online). The total product vibrational statedependent cross section as a function of the relative collision energy for $v^{\prime}=0-5$. The inset shows the corresponding theoretical relative populations of vibrational states taken from Candori [16].

single internal energy distribution, but it changes as a function of the scattering angle, as mentioned above. Finally, all Gaussians are multiplied with a step function with its edge at the kinematical cutoff. For the intervals in Figs. 1(b) and 1(c), the fits of the sum of Gaussians are shown as blue lines. The fitted heights and their standard deviation accuracies are used to determine the number of $\mathrm{N}_{2}^{+}$product ions scattered into a given vibrational level.

Figure 2 shows the number of ions in the vibrational states $v^{\prime}=1-6$ as a function of the scattering angle $\theta$ for all measured collision energies between 0.3 and $2 \mathrm{eV}$. For all energies vibrational excitation in the $v^{\prime}=1$ channel dominates in the forward direction whereas higher vibrational excitation is more prominent at larger scattering angles. With decreasing collision energy the products for each channel are scattered into increasingly larger scattering angles. A semiclassical surface hopping calculation cannot reproduce the angular dependence of the cross section at $1.7 \mathrm{eV}$, because the calculation predicts intensity out to much larger scattering angles than obtained in this work [30]. Qualitatively, the vibrational level dependence of the angular cross section agrees with the model presented by Candori et al. [16], who obtain the product vibrational levels from Landau-Zener curve crossing probabilities for vibrationally adiabatic intermolecular potentials. They suggest higher $v^{\prime}$ levels to be scattered into larger scattering angles, since the time the reaction complex spends at a particular curve crossing is increased with decreasing relative energy and thus decreasing impact parameter. In contrast to previous experiments [25], we do not see an enhancement of higher scattering angles at a particular relative energy and no backward scattered product ions. That implies that a scattering resonance is not likely to play an important role in this reaction system.

Figure 3 presents the vibrational branching ratios for $v^{\prime}=0$ to 5 , after integration over all scattering angles as a function of the collision energy. The values are normalized to unity for each energy. These integral data can be compared to previous studies of this reaction system. In contrast to the calculation by Candori et al. [16] (which is shown in the inset) we find higher vibrational excitation $\left(v^{\prime} \geq 2\right)$ to be more likely. While the calculation yields a ratio of $\left(v^{\prime}=2\right) /\left(v^{\prime}=1\right)$ of about $1 / 10$ for all scattering energies, we obtain a ratio of about $1 / 2$. This might be attributed to their employed ad hoc parameters for the coupling of the vibrational states, or may be caused by the semiclassical treatment of the problem. Liao et al. [23] measured the product vibrational excitation using chemical probing and found a ratio of $\left(\boldsymbol{v}^{\prime}=2\right) /\left(\boldsymbol{v}^{\prime}=1\right)$ of about $1 / 6$ at a collision energy of $1.2 \mathrm{eV}$. This is a significantly lower value compared to our measurement, which might be explained by a scattering-angle dependent acceptance probability in their experiment.

In conclusion, we have investigated the charge transfer reaction $\mathrm{Ar}^{+}+\mathrm{N}_{2} \rightarrow \mathrm{Ar}+\mathrm{N}_{2}$ between 0.3 and $2 \mathrm{eV}$ by the combination of crossed-beam techniques and $3 \mathrm{D}$ velocity map imaging. We have presented the detailed energy- and angle-differential cross sections for a range of collision energies. The achieved experimental resolution for ionmolecule reactive scattering has been improved significantly and gets closer to the resolution obtained in neutral-neutral reactions [39]. The vibrational state distributions show a clear increase in excitation for larger scattering angles. This increase becomes more prominent with decreasing collision energy for all kinematically allowed quantum states. These observations are qualitatively in line with calculations [16]. With some improvements in the calculations, a full quantitative agreement between experiment and theory, known for the neutral $\mathrm{H}+\mathrm{H}_{2}$ [40] and $\mathrm{F}+\mathrm{H}_{2}$ [39] reactions, now comes within reach for ion-molecule reactions as well.

This research has been supported by the Deutsche Forschungsgemeinschaft under Contract No. WE 2592/3-2 and by the EU Marie Curie Initial Training Network ICONIC. We thank the University of Freiburg, where the measurements presented here have been carried out, for supporting this research.

*roland.wester@uibk.ac.at

[1] J. J.H. Waite, D. T. Young, T.E. Cravens, A. J. Coates, F. J. Crary, B. Magee, and J. Westlake, Science 316, 870 (2007).

[2] M. Larsson, W.D. Geppert, and G. Nyman, Rep. Prog. Phys. 75, 066901 (2012).

[3] T. E. Cravens, Science 296, 1042 (2002).

[4] C. M. Lisse, D. J. Christian, K. Dennerl, K. J. Meech, R. Petre, H. A. Weaver, and S. J. Wolk, Science 292, 1343 (2001).

[5] E. A. Helder, J. Vink, C. G. Bassa, A. Bamba, J. A. M. Bleeker, S. Funk, P. Ghavamian, K. J. van der Heyden, F. Verbunt, and R. Yamazaki, Science 325, 719 (2009). 
[6] D. W. Savin, S. Krstic, Z. Haiman, and P.C. Stancil, Appita Journal : Journal of the Technical Association of the Australian and New Zealand Pulp and Paper Industry 606, L167 (2004).

[7] J. Perez, R. Olson, and P. Beiersdorfer, J. Phys. B 34, 3063 (2001).

[8] F. Rosmej, R. Stamm, and V. Lisitsa, Europhys. Lett. 73, 342 (2006).

[9] E. Wells, K. Carnes, B. Esry, and I. Ben-Itzhak, Phys. Rev. Lett. 86, 4803 (2001).

[10] C. Zipkes, S. Palzer, L. Ratschbacher, C. Sias, and M. Köhl, Phys. Rev. Lett. 105, 133201 (2010).

[11] S. Schmid, A. Härter, and J. H. Denschlag, Phys. Rev. Lett. 105, 133202 (2010).

[12] F. H. J. Hall and S. Willitsch, Phys. Rev. Lett. 109, 233202 (2012).

[13] A. W. Kleyn, V. N. Khromov, and J. Los, J. Chem. Phys. 72, 5282 (1980).

[14] P. Tosi, F. Eccher, D. Bassi, F. Pirani, D. Cappelletti, and V. Aquilanti, Phys. Rev. Lett. 67, 1254 (1991).

[15] Y.H. Huang, C.T. Rettner, D. J. Auerbach, and A. M. Wodtke, Science 290, 111 (2000).

[16] R. Candori, S. Cavalli, F. Pirani, A. Volpi, D. Cappelletti, P. Tosi, and D. Bassi, J. Chem. Phys. 115, 8888 (2001).

[17] L. Huewel, D. R. Guyer, G. H. Lin, and S. R. Leone, J. Chem. Phys. 81, 3520 (1984).

[18] M. Lipeles, J. Chem. Phys. 51, 1252 (1969).

[19] J.D. Kelley, G.H. Bearman, H.H. Harris, and J. J. Leventhal, Chem. Phys. Lett. 50, 295 (1977).

[20] W. Lindinger, F. Howorka, P. Lukac, S. Kuhn, H. Villinger, E. Alge, and H. Ramler, Phys. Rev. A 23, 2319 (1981).

[21] D. Smith and N. G. Adams, Phys. Rev. A 23, 2327 (1981).

[22] A.L. Rockwood, S. L. Howard, W.-H. Du, P. Tosi, W. Lindinger, and J.H. Futrell, Chem. Phys. Lett. 114, 486 (1985).

[23] C.-L. Liao, J.-D. Shao, R. Xu, G. D. Flesch, Y.-G. Li, and C. Y. Ng, J. Chem. Phys. 85, 3874 (1986).
[24] J.H. Futrell, Int. J. Quantum Chem. 31, 133 (1987).

[25] K. Birkinshaw, A. Shukla, S. L. Howard, and J. H. Futrell, Chem. Phys. 113, 149 (1987).

[26] D. M. Sonnenfroh and S. R. Leone, J. Chem. Phys. 90, 1677 (1989).

[27] S. L. Howard, Chem. Phys. Lett. 178, 65 (1991).

[28] M. R. Spalburg and E. A. Gislason, Chem. Phys. 94, 339 (1985).

[29] G. Parlant and E. A. Gislason, J. Chem. Phys. 86, 6183 (1987).

[30] E. E. Nikitin, M. Y. Ovchinnikova, and D. V. Shalashilin, Chem. Phys. 111, 313 (1987).

[31] D. C. Clary and D. M. Sonnenfroh, J. Chem. Phys. 90, 1686 (1989).

[32] R. Candori, F. Pirani, D. Cappelletti, P. Tosi, and D. Bassi, Int. J. Mass Spectrom. 223-224, 499 (2003).

[33] A. T. J. B. Eppink and D. H. Parker, Rev. Sci. Instrum. 68, 3477 (1997).

[34] J. Mikosch, U. Frühling, S. Trippel, D. Schwalm, M. Weidemüller, and R. Wester, Phys. Chem. Chem. Phys. 8, 2990 (2006).

[35] J. Mikosch, S. Trippel, C. Eichhorn, R. Otto, U. Lourderaj, J. X. Zhang, W. L. Hase, M. Weidemüller, and R. Wester, Science 319, 183 (2008).

[36] L. Pei and J. M. Farrar, J. Chem. Phys. 136, 204305 (2012).

[37] S. Trippel, M. Stei, R. Otto, P. Hlavenka, J. Mikosch, C. Eichhorn, U. Lourderarj, J. X. Zhang, W. L. Hase, M. Weidemüller, and R. Wester, J. Phys. Conf. Ser. 194, 012046 (2009).

[38] T. Kato, K. Tanaka, and I. Koyano, J. Chem. Phys. 77, 834 (1982).

[39] M. Qiu, Z. Ren, L. Che, D. Dai, S. A. Harich, X. Wang, X. Yang, C. Xu, D. Xie, M. Gustafsson, R. T. Skodje, Z. Sun, and D. H. Zhang, Science 311, 1440 (2006).

[40] S. A. Harich, D. Dai, C. C. Wang, X. Yang, S. D. Chao, and R. T. Skodje, Nature (London) 419, 281 (2002). 